\title{
Reconstrucción del Nervio Alveolar Inferior con Aloinjerto de Nervio Acelular Humano en Resección Mandibular
}

\author{
Reconstruction of the Inferior Alveolar Nerve with Human \\ Acellular Nerve Allograft in Mandibular Resection
}

\author{
Daniel Jeréz; Cristián Venables²; Germán Laissle³; Carlos Avendaño4 \& Humberto Velásquez ${ }^{5}$
}

JERÉZ, D.; VENABLES, C.; LAISSLE, G.; AVENDAÑO, C. \& VELÁSQUEZ, H. Reconstrucción del nervio alveolar inferior con aloinjerto de nervio acelular humano en resección mandibular. Int. J. Odontostomat., 14(3):400-406, 2020.

RESUMEN: La reconstrucción de nervios periféricos con aloinjertos nerviosos acelulares humanos en neurocirugía ha sido bastante estudiada estableciendo su predictibilidad y éxito en intervenciones principalmente en los nervios digitales de las manos. En cirugía maxilofacial existe una creciente investigación para poder restaurar el nervio alveolar inferior en cirugías de resección mandibular en donde la extirpación de esta estructura nerviosa es inevitable. El objetivo de esta publicación es mostrar un reporte de un caso en donde se realizó la reconstrucción del nervio alveolar inferior con aloinjerto de nervio acelular humano (Avance $®$ Nerve Graft, Axogen) con microcirugía para poder proveer de sensibilidad a la región de la cara afectada en un paciente reconstruido con un injerto de fíbula microvascularizada posterior a una hemimandibulectomía por ameloblastoma plexiforme.

PALABRAS CLAVE: nervio alveolar inferior, aloinjerto de nervio acelular humano, resección mandibular.

\section{INTRODUCCIÓN}

La resección parcial mandibular con márgenes de seguridad es el tratamiento habitual para los distintos tipos de ameloblastomas que afectan al cuerpo o la rama de la mandíbula (Effiom et al., 2018). Una vez hecha la resección se realiza la reconstrucción mandibular mediante el uso de fíbula microvascularizada que puede ser óseo u osteocutáneo en caso de ser requerido (HuentequeoMolina et al., 2018). Dentro de las secuelas más importantes de esta intervención se destaca la resección total del nervio alveolar inferior (NAI), que provee de sensibilidad a los dientes de la mandíbula, piel de la región mentoniana, el labio inferior y la encía vestibular de los dientes inferiores (Buch, 2011).

Sin duda la pérdida total de la sensibilidad de esta zona de la cara provoca un efecto negativo en la calidad de vida de las personas sometidas a este tipo de cirugías (van Gemert et al., 2015), por lo que vol- ver a establecer la función neurosensitiva resulta fundamental. Existen variadas técnicas de reposición o reconstrucción del NAI como los autoinjertos de nervio auricular mayor (Akbari \& Miloro, 2019) o autoinjerto de nervio sural (Miloro \& Stoner, 2005) que han reportado excelentes resultados demostrando su predictibilidad al recobrar la función sensorial, pero con la desventaja de producir morbilidad en el sitio donante (Ducic \& Yoon, 2019).

Los injertos de nervios autógenos actúan como andamiaje que no producen reacciones inmunológicas y suministran factores neurotróficos y células de Schwann importantes para la regeneración axonal (Ray \& Mackinnon, 2010). Por estas razones, este tipo de injertos se han convertido en el estándar de oro para el tratamiento de las lesiones nerviosas periféricas, sin embargo la cirugía para su obtención produce morbilidad en el sitio donante con pérdida de

\footnotetext{
${ }^{1}$ Cirujano Bucal y Maxilofacial, Clínica BUPA Santiago y Hospital de Niños Dr. Luis Calvo Mackenna, Santiago-Chile.

2 Cirujano Bucal y Maxilofacial, Práctica Privada.

${ }^{3}$ Cirujano Bucal y Maxilofacial, Clínica BUPA Santiago y Hospital Exequiel González Cortés, Santiago-Chile

${ }^{4}$ Profesor adjunto, Postgrado Cirugía Oral \& Maxilofacial, Facultad Odontología, Universidad San Sebastián, Lientur 1457, Concepción 4080871, Chile.

${ }^{5}$ Postgrado de Implantología, Facultad de Odontología, Universidad San Sebastián, Lientur 1457, Concepción 4080871, Chile.
} 
JERÉZ, D.; VENABLES, C.; LAISSLE, G.; AVENDAÑO, C. \& VELÁSQUEZ, H. Reconstrucción del nervio alveolar inferior con aloinjerto de nervio acelular humano en resección mandibular. Int. J. Odontostomat., 14(3):400-406, 2020.

sensibilidad, posibilidad de la formación de un neuroma, potencial dolor neuropático (Shanti \& Ziccardi, 2011), cicatrices excesivas y tiempo de operación prolongado (Ray \& Mackinnon).

Debido a ésto existe una creciente investigación orientada a utilizar aloinjertos nerviosos que en la última década han aumentado su popularidad, principalmente para la reparación de brechas nerviosas sensoriales cortas (Yampolsky et al., 2017). Dentro de sus ventajas incluyen su facilidad de uso, la falta de morbilidad del sitio donante y la disminución del tiempo operatorio (Rinker et al., 2017).

Uno de los más utilizados en las investigaciones es el aloinjerto de nervio acelular humano conocido por sus siglas en inglés como HANA (human acelular nerve allograft) (Mauch et al., 2019). En cuanto a sus propiedades se destaca que son acelulares y se desarrollaron en un esfuerzo por eliminar por completo la necesidad de utilizar medicamentos que provoquen inmunosupresión sistémica del paciente ( $\mathrm{Li}$ et al., 2015). Además, son tratados en un proceso denominado liofilización, en donde se elimina la humedad de los materiales a bajas temperaturas manteniendo su estructura, bioactividad y otras propiedades (Sheehan \& Liapis, 1998).

En relación con su composición presentan una matriz extracelular que consta de varias fibras de proteínas entrelazadas con diámetros que van desde decenas hasta cientos de nanómetros (Hudson et al., 2004). La estructura a nanoescala de esta matriz ofrece una red natural de nanofibras para soportar células y presentar un fondo para guiar el comportamiento celular (Zhu et al., 2016). Gracias a esta configuración sirven como un andamiaje nervioso seguro y efectivo, porque mantiene la mayor parte de la estructura natural y el microambiente del nervio periférico humano, sin provocar inmunogenicidad. Es así como se produce la migración desde los muñones nerviosos vecinos y se crea un entorno neurocompatible para fomentar la regeneración axonal. Además, se provoca una revascularización y repoblamiento de células del hospedero proporcionando así un entorno propicio para la regeneración (Brooks et al., 2012).

El objetivo de esta publicación es reportar un caso en donde se realizó la reconstrucción del nervio alveolar inferior con aloinjerto de nervio acelular humano (Avan-

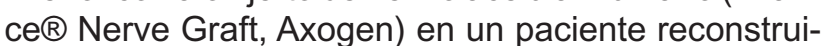
do con fíbula microvascularizada posterior a una hemimandibulectomía por ameloblastoma plexiforme.

\section{CASO CLÍNICO}

Paciente de 18 años, de sexo masculino, sin enfermedades sistémicas. Se presentó en la consulta con un aumento de volumen importante en el lado izquierdo de la cara, con abombamiento de tablas vestibular y lingual, movilidad de los dientes de la mitad izquierda de la mandíbula, dolor leve y sin compromiso neurosensitivo. La tomografía axial computarizada mostró una extensa lesión osteolítica multiloculada que comprometía el cuerpo y la rama mandibular izquierda, con expansión considerable y perforación de las corticales vestibular y lingual, además de rizalisis dentaria.

Se planificó una biopsia incisional y la colocación de cánulas de descompresión para esperar el resultado. En la histopatología de la biopsia se observaron varios fragmentos que presentaban extensa proliferación epitelial dispuesta en cordones entrelazados, con células en la periferia cúbicas o columnares, hacia el centro algunas estrelladas o alargadas, pero muchas de núcleos ovalados, con escasas mitosis típicas, y entre ellas tejido conjuntivo laxo, con pequeños vasos y escaso infiltrado mononuclear arrojando el diagnóstico de Ameloblastoma Plexiforme.

Se decidió realizar las exodoncias de los dientes inferiores izquierdos para permitir el cierre mucoso 3 meses previo a la cirugía reconstructiva y así evitar la exposición del injerto. Luego de esperar el tiempo estimado se planificó la cirugía resectiva y reconstructiva mediante planificación virtual. Se solicitaron una tomografía computarizada (TC) maxilofacial con cortes menores a $1 \mathrm{~mm}$ para la planificación virtual e impresión de un modelo esterolitográfico y además una TC de extremidades para la elección del colgajo, orientación y adaptación mediante planificación virtual e impresión de modelos para la adaptación de una placa de reconstrucción. Mediante el uso del software Mimmics se realizó la planificación de la hemimandibulectomía entre los dientes 3.3 y 3.4 con desarticulación ipsilateral y la reconstrucción con injerto microvascular de fíbula izquierda. Por otra parte, en el software 3-matic se realizó el diseño de las guías de corte y posicionamiento del injerto de fíbula para reconstruir el defecto. Luego se utilizó la impresora Stratasys Eden 260 donde se imprimieron las guías de corte para mandibulectomía, guía de reposición de injerto de fíbula izquierdo y modelo estereolitográfico con injerto adaptado para realizar el pre-contorneo y elección de la placa de osteosíntesis 
de reconstrucción. Se decidió utilizar una placa de reconstrucción sistema 2.4 Enterfix con sistema Locking. Además, se realizó una planificación virtual para la reconstrucción del nervio alveolar inferior midiendo el diámetro y largo que se considera incluido en la resección. Se planificó la reconstrucción del nervio con aloinjerto de nervio acelular humano Avance de $70 \mathrm{~mm}$ de largo y 2-3 mm de diámetro (Fig. 1).

Bajo anestesia general e intubación nasotraqueal, se realizó un abordaje preauricular izquierdo con modificación endaural, se accedió a la articulación temporomandibular de ese mismo lado y se liberó el cóndilo mandibular manteniendo el disco articular. Luego se alcanzó la lesión mediante una cervicotomía izquierda, preparando los vasos cervicales para la posterior microanastomosis vascular del injerto. Se decoló el cuerpo y la rama mandibular, observándose la extensión del tumor. La sección, preparación y reconstrucción del nervio fue realizada por Daniel Jerez DDS, OMFS. Se realizó la sección del nervio alveolar cefálico a la língula y el nervio mentoniano con tijera de microcirugía y se preparó para la posterior reconstrucción. Se marcaron ambos cabos, proximal y distal, con punto de nylon $5 / 0$ para posterior localización. Se instaló la guía de corte anterior y se realizó la osteotomía entre los dientes 3.3 y 3.4. Se realizó la hemimandibulectomía con desarticulación según la planificación y se instaló la placa de osteosíntesis pre-contorneada. Mediante el uso de un microscopio (microscopio ZEISS modelo OPMI VISU 160 bajo magnificación de 10 X) se realizó la preparación de los cabos distal y proximal de nervio alveolar y mentoniano. Se observó una sección plana e indemnidad de los fascículos para poder realizar la reconstrucción. Se solicitó la preparación del injerto según indicaciones del fabricante. El injerto se colocó en suero fisiológico para su descongelación (Fig. 2). Bajo microscopio se efectuó la microcoaptación primaria

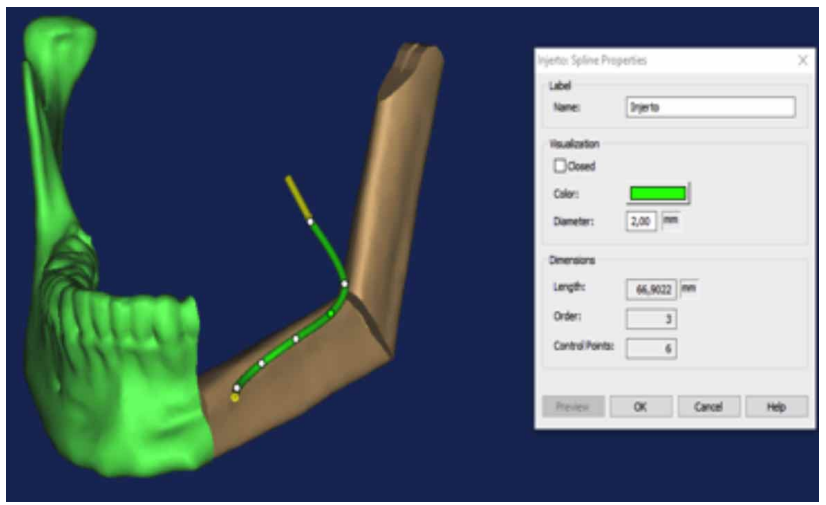

Fig. 1. Planificación virtual del injerto del NAI. proximal entre el nervio alveolar y el injerto con 6 puntos discontinuos de Ethilon 8/0 epineurales (Figs. 3, 4 y 5). Posteriormente la microcoaptación distal con 6 puntos discontinuos de nylon $8 / 0$ entre injerto y el nervio mentoniano. El injerto alveolar se suspende con lazo de Vicryl 4/0 hacia el colgajo cervical para evitar su daño durante la manipulación y adaptación del colgajo de fíbula. De forma simultánea, la cirujana reconstructiva (A.G) realizó la toma de fíbula microvascular de pierna izquierda, sin paleta cutánea, mediante técnica tradicional. Se instalaron guías de corte para proceder a la preparación del colgajo y su posterior colocación en el sitio de la lesión resecada (Fig. 6). Luego se instaló la placa de reconstrucción y se realizó la microanastomosis venosa con 2 puntos

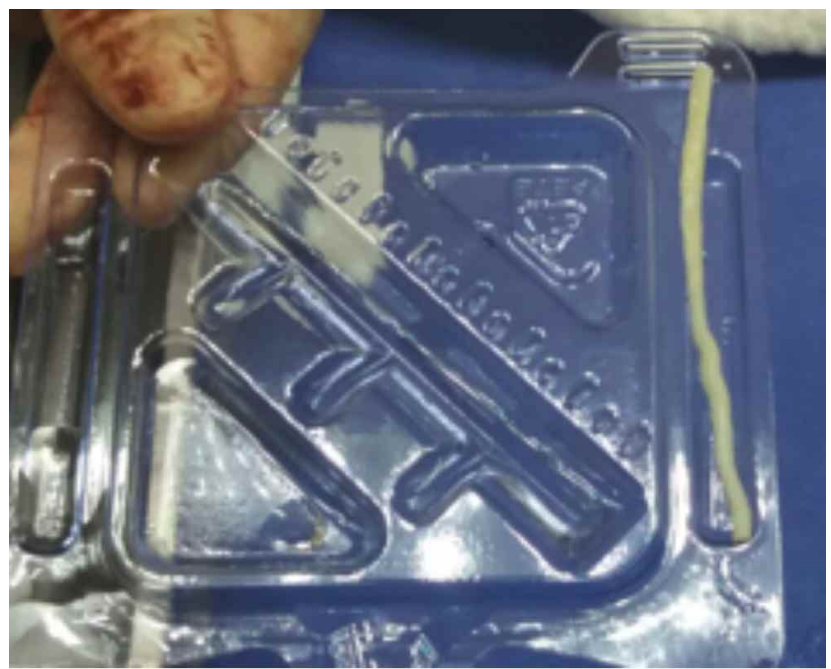

Fig. 2. Avance ${ }^{\circledR}$ Nerve Graft, Axogen.

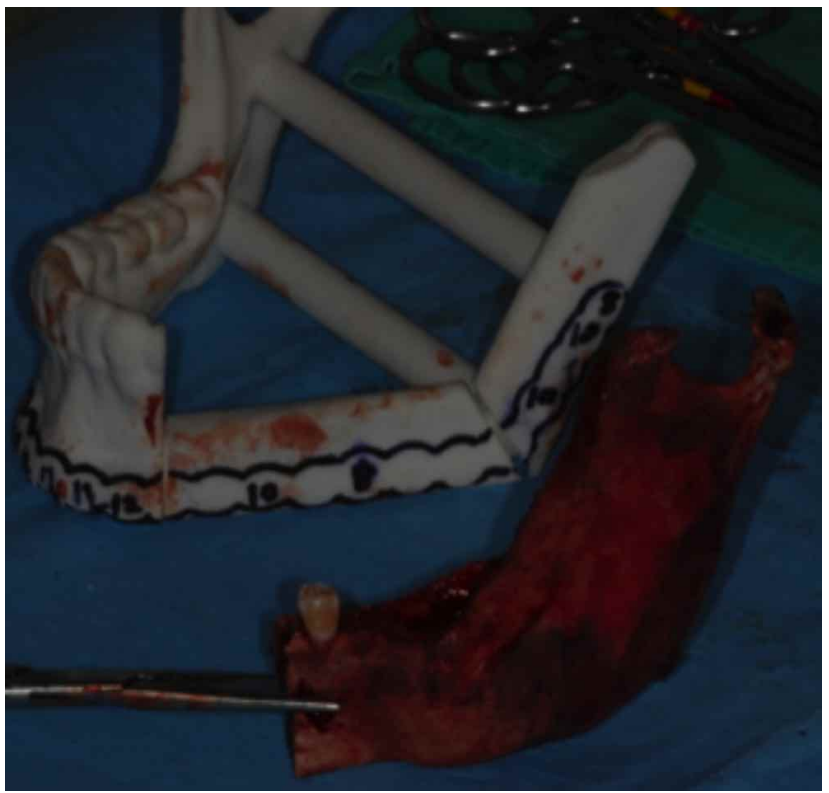

Fig. 3. Resección Mandibular. 


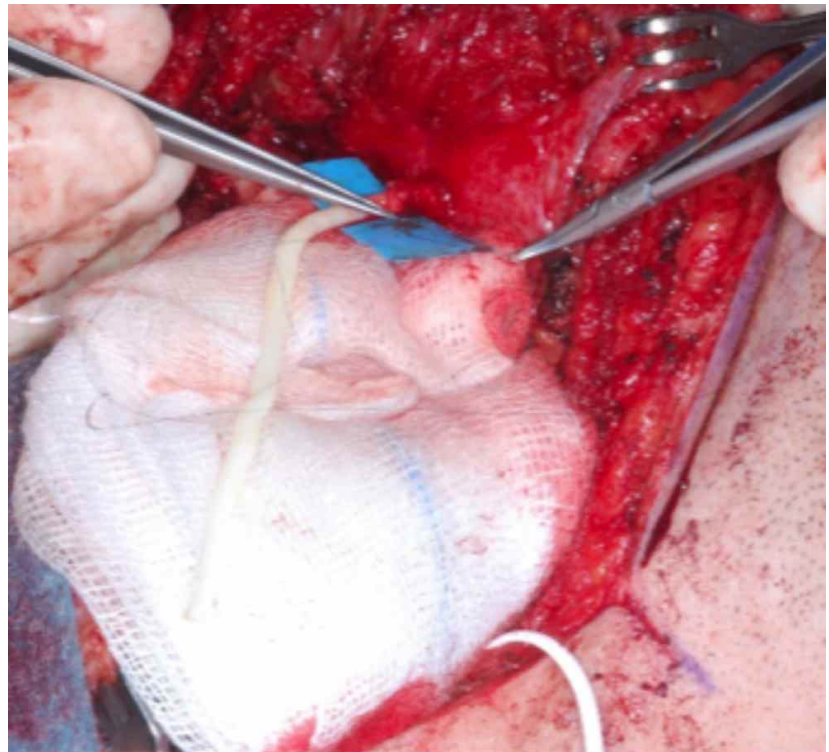

Fig. 4. Aloinjerto de nervio acelular humano.

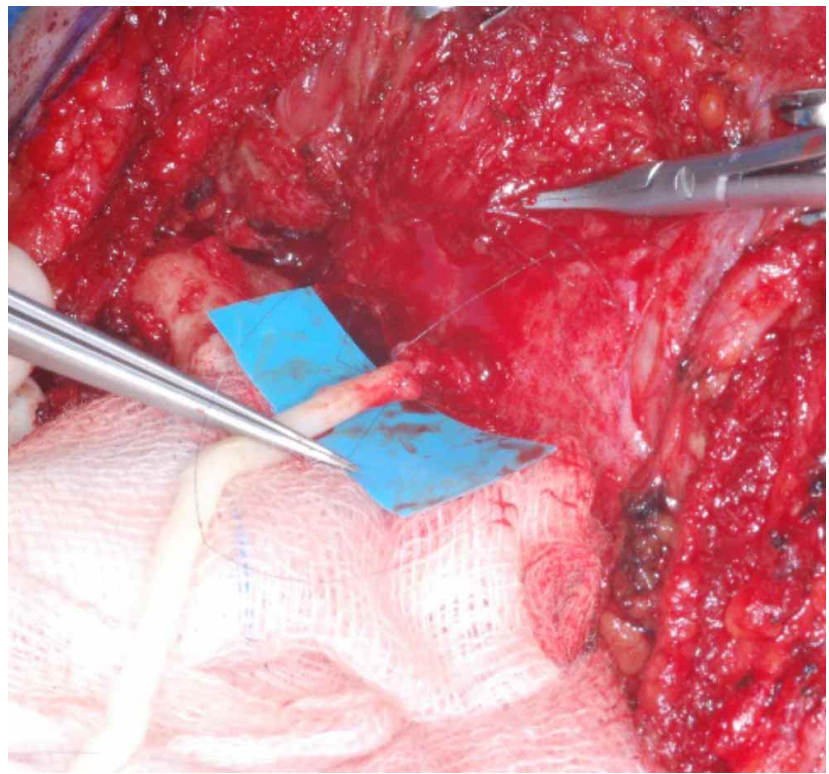

Fig. 5. Microcoaptación primaria entre injerto y nervio mentoniano con puntos discontinuos de ethilon $8 / 0$. continuos de ethilon 9/0 y la microanastomosis arterial con puntos discontinuos de ethilon 9/0 (Fig. 7). Se comprobó el flujo y retorno sanguíneo del injerto y se cerraron ambos abordajes faciales y de la fíbula por planos. El paciente se trasladó a la Unidad de Paciente Crítico extubado permaneciendo dos días y luego a una habitación tradicional. Fue dado de alta a los 5 días postoperatorio y controlado a los 3 días, evolucionando satisfactoriamente.

Para el control de seguimiento del injerto alveolar se realizaron test de sensibilidad postoperatorios seriados al mes, tercer y sexto mes post quirúrgico. El test realizado consistió en un algoritmo de 3 niveles (Nivel A, B y C) publicado por Zuniga et al. (1998), considerado como el estándar de oro en el diagnóstico y seguimiento de lesiones de nervio trigémino. Al sexto mes post operatorio el paciente presentó una recuperación completa de la sensibilidad del labio inferior, clasificado como una S4 según la clasificación de Medical Research Council Scale (Resultados paciente: Discriminación de localización 15 de 15 y Discriminación de 2 puntos promedio 3 intentos de $6 \mathrm{~mm}$ ).

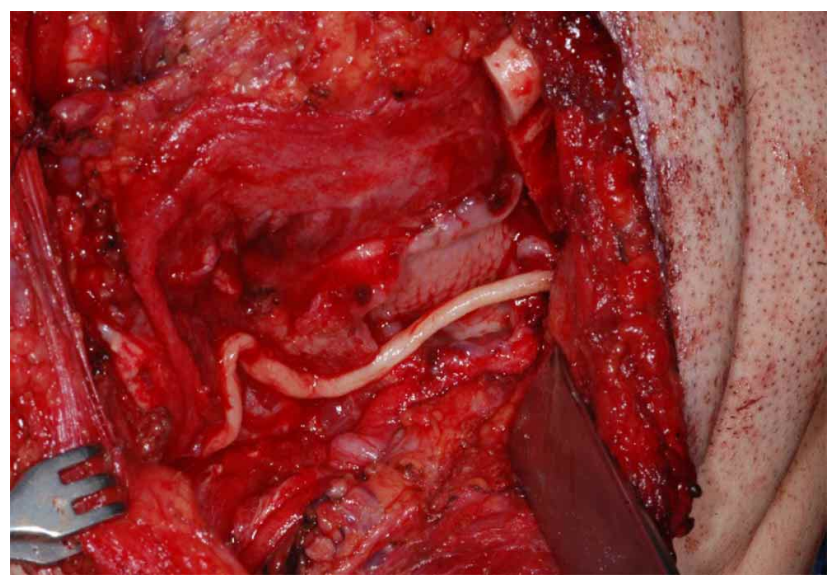

Fig. 6. Injerto en posición con ambas coaptaciones realizadas.
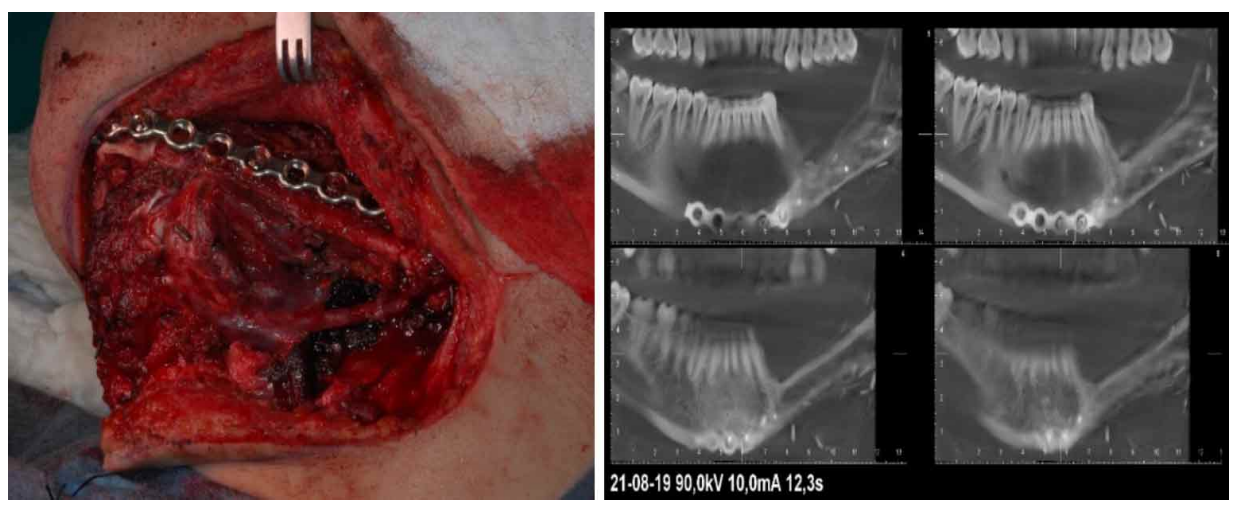

Fig. 7. Reconstrucción mandibular con injerto de fíbula y placa de reconstrucción. Injerto alveolar con lazo de vicryl suspendido en el colgajo cervical para evitar su daño durante la manipulación y adaptación del colgajo de fíbula. 


\section{DISCUSIÓN}

La reconstrucción de nervios periféricos con Aloinjerto de nervio acelular humano ha sido bastante estudiada sobre todo en los nervios digitales de las manos (Mauch et al.). En este sentido, Brooks et al. demostraron en un estudio multicéntrico sobre aloinjertos nerviosos procesados (Avance ${ }^{\circledR}$ Nerve Graft, AxoGen, Inc) en donde 25 cirujanos aportaron datos de 132 lesiones de nervios periféricos individuales con una recuperación significativa en el $87 \%$ de las reparaciones concluyendo que los aloinjertos nerviosos procesados funcionaron bien y resultaron ser seguros y efectivos en defectos nerviosos sensoriales, mixtos y motores de entre $5 \mathrm{~mm}$ y $50 \mathrm{~mm}$ de longitud.

De igual forma Azouz et al. (2018) realizaron una encuesta a 461 cirujanos de distintas especialidades concluyendo que el aloinjerto de nervio acelular humano supera al conector nervioso como el gold standard debido a que casi el $70 \%$ de los cirujanos de mano lo utilizan en su práctica y un mayor porcentaje de encuestados lo eligieron como su primera opción para los procedimientos de reparación nerviosa en comparación con el conector, el autoinjerto nervioso o el injerto venoso. Ante esta evidencia, las investigaciones en cirugía maxilofacial y de cabeza y cuello se orientan en determinar la efectividad de la utilización del aloinjerto de nervio acelular humano en la reconstrucción del NAI. Existen variadas investigaciones que reportan reconstrucción inmediata del NAI con este tipo de injerto en defectos de pequeño a largo alcance (Shanti \& Ziccardi; Zuniga, 2015; Zuñiga et al., 2017; Yampolsky et al.).

En defectos pequeños, Yampolsky et al. realizaron un estudio con 16 pacientes (12 mujeres y 4 hombres) que tuvieron una lesión del NAI (8 pacientes) o el nervio lingual (8 pacientes) y cuyo mecanismo más común de lesión fue la extracción del tercer molar ( $n=9 ; 56,25 \%)$. De los 16 pacientes, $15(93,75$ $\%)$ lograron una recuperación sensorial funcional durante el período de estudio determinando que sus resultados respaldan la hipótesis de que los aloinjertos nerviosos procesados son efectivos para reconstruir defectos nerviosos pequeños $(<2 \mathrm{~cm})$ del nervio trigémino.

Asimismo, Zuniga desarrolló un análisis retrospectivo del uso de aloinjerto de nervio acelular hu- mano (Avance ${ }^{\circledR}$ Nerve Graft, AxoGen, Inc) para la reconstrucción del nervio lingual y el NAI en 26 sujetos con 28 injurias y realizó evaluaciones sensoriales (sensación de trazo de pincel, discriminación estática de 2 puntos, detección de contacto, presión, umbral de dolor y tolerancia al dolor por presión) antes de la operación y 3,6 , y 12 meses después de la reconstrucción quirúrgica usando una escala clínica de pruebas neurosensoriales concluyendo que el 87 $\%$ había mejorado puntuaciones neurosensoriales sin experiencias adversas reportadas y reportó porcentajes de niveles de mejoría similares para ambos nervios (87 \% para nervio lingual y $88 \%$ para el NAI).

Por otra parte, Shanti \& Ziccardi también lo utilizaron en un reporte de caso de una mujer de 62 años sometida a reconstrucción de un segmento del NAl debido a una injuria producida por una obliteración del canal mandibular con injerto óseo para una preservación alveolar posterior a una exodoncia del diente 3.6. Ellos reportaron que a los 5 meses después de la cirugía, el paciente informó hormigueo y picazón en el lado afectado de la barbilla y estaba satisfecho con la mejora sensorial.

La reconstrucción de defectos en el territorio maxilofacial sigue siendo un gran desafío para los cirujanos, quienes han intentado realizar reconstrucciones mandibulares por más de un siglo (Alister et al., 2017). El siguiente reto ha sido determinar la efectividad de la reconstrucción del NAI en lesiones de largo alcance y en este sentido Salomon et al.,(2016) investigaron a 7 pacientes de los cuales 6 se reconstruyeron con un aloinjerto nervioso (Avance ${ }^{\circledR}$ Nerve Graft, AxoGen, Inc) de $7 \mathrm{~cm}$ y 1 defecto nervioso se reconstruyó con un injerto de $5 \mathrm{~cm}$ en donde el 85,7 $\%$ de los pacientes mostraron un retorno leve de dolor superficial y sensación táctil sin una respuesta exacerbada y sólo 1 de los 7 pacientes no tuvo recuperación neurosensorial, infiriendo que la reconstrucción inmediata de la NAl con injerto nervioso alogénico de defectos de largo alcance mayores a 5 $\mathrm{cm}$ es una opción viable y predecible.

En este sentido, Zuniga et al. (2017) evaluaron defectos nerviosos aún más largos $(62,7 \mathrm{~mm}$ en promedio) y realizaron una investigación para determinar si la reconstrucción inmediata del nervio alveolar inferior con un aloinjerto de nervio acelular humano en conjunto con la reconstrucción de la mandíbula sería efectiva para restaurar de manera segura la sensación subjetiva y lograr la recuperación neurosensorial funcional. Ellos analizaron 18 
JERÉZ, D.; VENABLES, C.; LAISSLE, G.; AVENDAÑO, C. \& VELÁSQUEZ, H. Reconstrucción del nervio alveolar inferior con aloinjerto de nervio acelular humano en resección mandibular. Int. J. Odontostomat., 14(3):400-406, 2020.

pacientes y los datos se obtuvieron antes de la cirugía y a los 3,6 y 12 meses después reportando que el $90 \%$ de los pacientes lograron una recuperación sensorial funcional e informaron sensaciones similares a los valores subjetivos preoperatorios determinando que está técnica es efectiva y segura cuando se inserta inmediatamente el injerto nervioso con la resección y reconstrucción de la mandíbula. Estos resultados son comparables a los obtenidos por nosotros en este caso clínico.

A pesar de que la limitada evidencia confirma lo predecible y exitoso que puede ser esta técnica en lesiones de $70 \mathrm{~mm}$ de longitud (Safa \& Buncke, 2016) la mayoría de los cirujanos en el pasado no han abordado la reconstrucción microquirúrgica del $\mathrm{NAl}$ en el momento de la resección mandibular ya sea de manera inmediata o diferida. En este sentido, en una encuesta electrónica en línea realizada a cirujanos de cabeza y cuello donde se consultaban los patrones de práctica y las opiniones subjetivas sobre el injerto del nervio alveolar inferior en el momento de la resección mandibular sólo el 10,6 $\%$ de los encuestados realizó la reconstrucción de la NAI "siempre" en una resección mandibular por tumores benignos, mientras que sólo el 1,1\% la realizó siempre en una resección mandibular por tumores malignos. Entre los 93 participantes, las barreras de utilización más importantes fueron la falta de evidencia para mejorar la calidad de vida, el impacto potencial de la radiación en la curación de los nervios y la falta de eficacia para la restauración de la función neurosensorial (Akbari \& Miloro). Al evaluar estos datos resulta insólito que a pesar de que la literatura basada en evidencia muestra avances importantes del injerto de NAI inmediato con resección mandibular en tumores benignos, la mayoría de los cirujanos de cabeza y cuello no realizan este procedimiento de manera rutinaria.

La resección mandibular se ha asociado con una mala calidad de vida relacionada con la salud oral (CVRSO), en este sentido Yang et al. (2014) evaluaron la CVRSO en relación a las reconstrucciones que se realizan de la mandíbula con injerto libre de fíbula microvascularizada aplicando los cuestionarios UW-QOL y el OHIP-14 después de 12 meses de postquirúrgicos concluyendo que este tipo de reconstrucciones afectan significativamente en la calidad de vida y las funciones orales de los pacientes, por lo que si se hiciera una reconstrucción de nervio alveolar se podría mejorar la sensibilidad y por lo tanto la calidad de vida.
Las personas sufren por los problemas estéticos evidentes que producen una baja en su autoestima y en las relaciones sociales que mantienen (Li et al., 2014), además presentan problemas funcionales de masticación que les impide disfrutar de los alimentos y reducen su dieta a la ingesta de comida que pueden consumir, una mejor en la percepción de la sensibilidad en los segmentos afectados de la cara podría mejorar su calidad de vida por lo que los cirujanos deberían considerar realizar la reconstrucción del NAI (Petrovic et al., 2019).

\section{CONCLUSIÓN}

La reconstrucción del NAl en resecciones mandibulares es un procedimiento que ha reportado excelentes resultados, pero existe la necesidad clara de realizar ensayos clínicos controlados aleatorizados para determinar su real efectividad en la recuperación de la sensibilidad de la zona afectada de la cara. La investigación futura no debería centrarse solo en el logro de la recuperación sensorial funcional, sino también en el impacto de los indicadores de calidad de vida que tienen los pacientes para poder mejorarlos y obtener un compromiso del paciente que influya positivamente en su rehabilitación.

JERÉZ, D.; VENABLES, C.; LAISSLE, G.; AVENDAÑO, C. \& VELÁSQUEZ, H. Reconstruction of the inferior alveolar nerve with human acellular nerve allograft in mandibular resection: Case report. Int. J. Odontostomat., 14(3):400-406, 2020.

ABSTRACT: The reconstruction of peripheral nerves with allografts of human acellular nerves in neurosurgery is well studied, establishing its predictability and success in interventions mainly in the digital nerves of the hands. In maxillofacial surgery there is a growing investigation to be able to restore the inferior alveolar nerve in mandibular resection surgeries where the removal of this nervous structure is inevitable. The objective of this publication is to show a case report in which the reconstruction of the inferior alveolar nerve was performed with human acellular nerve allograft (Avance $®$ Nerve Graft, Axogen) with microsurgery in order to provide sensitivity to the region of the affected face in a reconstructed patient with a microvascularized bone fibula graft after hemimandibulectomy due to plexiform ameloblastoma.

KEY WORDS: inferior alveolar nerve, human acellular nerve allograft, resección mandibular resection. 


\section{REFERENCIAS BIBLIOGRÁFICAS.}

Akbari, M. \& Miloro, M. The inferior alveolar nerve: to graft or not to graft in ablative mandibular resection? J. Oral Maxillofac. Surg., 77(6):1280-5, 2019.

Alister, J. P.; Veuthey, C.; Uribe, F.; Vásquez, B.; del Sol, M. \& Olate, $\mathrm{S}$. Experimental model for the study of mandibular reconstruction. Options in Rabbits Oryctolagus cuniculus. Int. J. Morphol., 35(3):1185-90, 2017

Azouz, S. M.; Lucas, H. D.; Mahabir, R. C. \& Noland, S. S. A survey of the prevalence and practice patterns of human acellular nerve allograft use. Plast. Reconstr. Surg. Glob. Open, 6(8):e1803, 2018.

Brooks, D.; Weber, R. V.; Chao, J. D.; Rinker, B. D.; Zoldos, J.; Robichaux, M. R.; Ruggeri, S. B.; Anderson, K. A.; Bonatz, E. E.; Wisotsky, S. M.; et al. Processed nerve allografts for peripheral nerve reconstruction: a multicenter study of utilization and outcomes in sensory, mixed, and motor nerve reconstructions. Microsurgery, 32(1):1-14, 2012.

Buch, H. A. Clinical anatomy of inferior alveolar nerve block anesthesia. Clin. Anat., 24(4):515-7, 2011.

Ducic, I. \& Yoon, J. Reconstructive options for inferior alveolar and lingual nerve injuries after dental and oral surgery: an evidencebased review. Ann. Plast. Surg., 82(6):653-60, 2019.

Effiom, O. A.; Ogundana, O. M.; Akinshipo, A. O. \& Akintoye, S. O. Ameloblastoma: current etiopathological concepts and management. Oral Dis., 24(3):307-16, 2018.

Hudson, T. W.; Liu, S. Y. \& Schmidt, C. E. Engineering an improved acellular nerve graft via optimized chemical processing. Tissue Eng., 10(9-10):1346-58, 2004.

Huentequeo-Molina, C.; Pino-Díaz, DD.; Moreno-Apablaza, E.; Alister, J. P.; Uribe, F.; Unibazo-Zuñiga, A.; Miño, M.; Ostrosky, A. \& Olate, S. Microvascularized flaps in maxillofacial reconstruction: advances in microsurgery. Int. J. Odontostomat., 12(3):309-19, 2018.

Li, X. Y.; Hu, H. L.; Fei, J. R.; Wang, X.; Wang, T. B.; Zhang, P. X. \& Chen, $H$. One-stage human acellular nerve allograft reconstruction for digital nerve defects. Neural Regen. Res., 10(1):95-8, 2015.

Li, X.; Zhu, K.; Liu, F. \& Li, H. Assessment of quality of life in giant ameloblastoma adolescent patients who have had mandible defects reconstructed with a free fibula flap. World J. Surg. Oncol., 12:201, 2014.

Mauch, J. T.; Bae, A. Shubinets, V. \& Lin, I. C. A systematic review of sensory outcomes of digital nerve gap reconstruction with autograft, allograft, and conduit. Ann. Plast. Surg., 82(4S Suppl. 3):S247-S255, 2019.

Miloro, M. \& Stoner, J. A. Subjective outcomes following sural nerve harvest. J. Oral Maxillofac. Surg., 63(8):1150-4, 2005.

Petrovic, I.; Panchal, H.; De Souza Franca, P. D.; Hernandez, M.; McCarthy, C. C. \& Shah, J. P. A systematic review of validated tools assessing functional and aesthetic outcomes following fibula free flap reconstruction of the mandible. Head Neck, 41(1):24855,2019

Ray, W. Z. \& Mackinnon, S. E. Management of nerve gaps: Autografts, allografts, nerve transfers, and end-to-side neurorrhaphy. Exp. Neurol., 223(1):77-85, 2010.

Safa, B. \& Buncke, G. Autograft substitutes: conduits and processed nerve allografts. Hand Clin., 32(2):127-40, 2016.

Salomon, D.; Miloro, M. \& Kolokythas, A. Outcomes of immediate allograft reconstruction of long-span defects of the inferior alveolar nerve. J. Oral Maxillofac. Surg., 74(12):2507-14, 2016.

Shanti, R. M. \& Ziccardi, V. B. Use of decellularized nerve allograft for inferior alveolar nerve reconstruction: a case report. J. Oral Maxillofac. Surg., 69(2):550-3, 2011.
Sheehan, P. \& Liapis, A. I. Modeling of the primary and secondary drying stages of the freeze drying of pharmaceutical products in vials: Numerical results obtained from the solution of a dynamic and spatially multi-dimensional lyophilization model for different operational policies. Biotechnol. Bioeng., 60(6):712-28, 1998.

van Gemert, J.; Holtslag, I.; van der Bilt, A.; Merkx, M.; Koole, R. \& Van Cann, E. Health-related quality of life after segmental resection of the lateral mandible: Free fibula flap versus plate reconstruction. J. Craniomaxillofac. Surg., 43(5):658-62, 2015.

Yampolsky, A.; Ziccardi, V. \& Chuang, S. K. Efficacy of acellular nerve allografts in trigeminal nerve reconstruction. J. Oral Maxillofac. Surg., 75(10):2230-4, 2017.

Yang, W.; Zhao, S.; Liu, F. \& Sun, M. Health-related quality of life after mandibular resection for oral cancer: reconstruction with free fibula flap. Med. Oral Patol. Oral Cir. Bucal, 19(4):e414-8, 2014.

Zhu, S.; Zhu, Q.; Liu, X.; Yang, W.; Jian, Y.; Zhou, X.; He, B.; Gu, L.; Yan, L.; Lin, T.; et al. Three-dimensional reconstruction of the microstructure of human acellular nerve allograft. Sci. Rep., 6:30694, 2016.

Zuniga, J. R. Sensory outcomes after reconstruction of lingual and inferior alveolar nerve discontinuities using processed nerve allograft--a case series. J. Oral Maxillofac. Surg., 73(4):734-44, 2015.

Zuniga, J. R.; Meyer, R. A.; Gregg, J. M.; Miloro, M. \& Davis, L. F. The accuracy of clinical neurosensory testing for nerve injury diagnosis. J. Oral Maxillofac. Surg., 56(1):2-8, 1998.

Zuniga, J. R.; Williams, F. \& Petrisor, D. A case-and-control, multisite, positive controlled, prospective study of the safety and effectiveness of immediate inferior alveolar nerve processed nerve allograft reconstruction with ablation of the mandible for benign pathology. J. Oral Maxillofac. Surg., 75(12):2669-81, 2017.

Dirección para correspondencia:

Carlos Avendaño Mendoza

Facultad Odontología

Universidad San Sebastián

Lientur \#1457

Concepción

CHILE

Email: cavendanom@docente.uss.cl

Recibido : 25-12-2019

Aceptado: $31-03-2020$ 